\title{
BMI open Aerobic neuromuscular electrical stimulation - an emerging technology to improve haemoglobin A1c in type 2 diabetes mellitus: results of a pilot study
}

\author{
Louis Crowe, ${ }^{1}$ Brian Caulfield ${ }^{2}$
}

To cite: Crowe L, Caulfield B. Aerobic neuromuscular electrical stimulation-an emerging technology to improve haemoglobin $\mathrm{A} 1 \mathrm{c}$ in type 2 diabetes mellitus: results of a pilot study. BMJ Open 2012;2:e000219. doi:10.1136/

bmjopen-2011-000219

- Prepublication history for this paper is available online. To view this file please visit the journal online (http://dx. doi.org/10.1136/ bmjopen-2011-000219).

Received 16 June 2011 Accepted 11 January 2012

This final article is available for use under the terms of the Creative Commons Attribution Non-Commercial 2.0 Licence; see http://bmjopen.bmj.com

\footnotetext{
${ }^{1}$ Institute of Sport and Health, Newstead Building, University College Dublin, Dublin, Ireland

${ }^{2}$ Department of

Physiotherapy, Health Sciences Building, University College Dublin, Dublin, Ireland
}

Correspondence to Dr Louis Crowe; louiscrowe@gmail.com

\section{ABSTRACT}

Objectives: A new generation of neuromuscular electrical stimulation (NMES) devices can exercise aerobically at equivalent rates to voluntary exercise. Many with type 2 diabetes cannot or will not exercise sufficiently. The objective of this pilot investigation was to see (1) if it was an acceptable training modality for men with type 2 diabetes mellitus and (2) to assess effects on haemoglobin A1c levels.

Design, setting, participants and intervention: $A$ case series of eight men with type 2 diabetes mellitus (aged $53 \pm 8$; body mass index $32 \pm 55 \mathrm{~kg} / \mathrm{m}^{2}$ ) trained with the NMES system for $1 \mathrm{~h} 6$ times weekly for 8 weeks, unsupervised, at home. There were no other medication or lifestyle interventions. The aerobic NMES exercise system delivers a repeating set of four complex staggered pulses at high intensities (typically $100 \mathrm{~mA}+$ ) through an array of eight thigh electrodes.

Outcome measures: The primary outcome measures were changes in haemoglobin $\mathrm{A} 1 \mathrm{C}$ and the responses in a questionnaire on participants' perceptions of the system. Body mass and composition were also measured before and after the NMES intervention period.

Results: All participants could use the system at a level that left them breathless and sweaty and with a heart rate over 120 beats per minute. Haemoglobin A1c levels improved by $0.8 \pm 0.7 \%$ from $7.4 \pm 1.3 \%$ (mean \pm SD) to $6.6 \pm 1.0 \%(p=0.01)$. All participants considered the system suitable for people with diabetes, would recommend it and would continue to use it twice a week 'to maintain improvements'.

Conclusions: These results suggest that aerobic NMES may be acceptable and have a beneficial effect on haemoglobin A1c of some men with diabetes. The treatment may be of particular benefit in those who will not or cannot do adequate amounts of voluntary exercise. A randomised control trial is required for conclusive efficacy data.

\section{INTRODUCTION}

A new generation of neuromuscular electrical stimulation (NMES) devices can exercise aerobically at equivalent rates to

\section{ARTICLE SUMMARY}

Article focus

- Advanced NMES techniques can now deliver aerobic exercise at training intensities. Improvements in aerobic fitness have been shown in the healthy, those with cardiac failure and the obese.

- It was hypothesised that this could be of benefit to those with type 2 diabetes, particularly those with barriers to voluntary exercise.

- A pilot study was undertaken to assess the system and its effects on $\mathrm{HbA1C}$.

Key messages

- All participants could use the system, unsupervised, at home, at intensities that made them sweaty and breathless.

- Average improvement in $\mathrm{HbA1c}$ of 0.8 $\pm 0.7 \%(p=0.01)$ is consistent with exercise interventions.

- The system may be an alternative for patients that will not or cannot undertake voluntary exercise.

Strengths and limitations of this study

- The technology used is now well proven to have substantial aerobic training effects in other groups.

- There were no other lifestyle interventions.

- This is a small uncontrolled pilot study on a group of men who pro-actively volunteered for participation in an exercise programme. While the results are promising, it was not a randomised controlled trial, and the sample may not be representative of patients with type 2 diabetes mellitus.

voluntary exercise. ${ }^{1-5}$ As many people with type 2 diabetes cannot or will not exercise sufficiently, a case series was undertaken to assess the acceptability and efficacy of treatment with this new exercise modality in a male type 2 diabetic population. There is consensus among the major diabetes organisations on the importance of physical activity in the prevention and treatment of type 2 diabetes. ${ }^{6}{ }^{7}$ However, there are many 
real and perceived barriers to exercise in this group ${ }^{8}$ including obesity. ${ }^{9}$ There are those who cannot exercise, for example, at an extreme people with spinal cord injuries. There are those who have relative barriers to exercise, commonly due to musculoskeletal conditions, particularly arthritis. People with arthritis exercise less ${ }^{10}$; there are 21.1 million US adults with arthritis-attributable activity limitation. ${ }^{11}$ There are over 10 million in the USA with diabetes and arthritis. ${ }^{12}$ Additionally, there are those who could but will not exercise sufficiently, perhaps due to the commitment required, particularly time commitment, or a host of psychological or other barriers. ${ }^{13}$ De facto, standard exercise regimes are insufficiently appealing to the many who could but do not regularly exercise. ${ }^{14}$

NMES has been used for many decades to elicit muscle contractions with an electrical impulse. A sophisticated NMES device (NT2010 Research Stimulator; Bio-Medical Research Ltd, Galway, Ireland) seems to offer a genuine alternative to vigorous voluntary aerobic exercise. It can deliver complex pulses in unusual patterns that seem to be well tolerated at high intensities (up to $200 \mathrm{~mA}$ ). A technical description of the system is given below; at the time of writing, they are still prototype devices. Previous studies have demonstrated that using this, NMES subjects can increase maximum aerobic capacity $\left(\mathrm{VO} 2_{\max }\right)$, typically by around $10 \%$ over a $6-8$ week training period. This has been shown in the comparatively fit, ${ }^{2}$ those with cardiac failure, ${ }^{3}$ the sedentary ${ }^{4}$ and in the patients with obesity. At aggressive intensities $(160-200 \mathrm{~mA})$, it has been shown to induce a maximal heart rate while exercising at over $75 \%$ of $\mathrm{VO} 2_{\text {max }}{ }^{1}$ Even traditional NMES techniques exercising at just two metabolic equivalents (oxygen consumption of $7 \mathrm{ml}$ / $\mathrm{kg} / \mathrm{min}$ ) has been shown to enhance total body glucose uptake and attenuate postprandial hyperglycemia in the obese. ${ }^{15}$ The therapeutic potential of traditional NMES on diabetes has been investigated previously with no improvement in haemoglobin A1c (HbAlc) or insulin sensitivity. ${ }^{16}$ However, as a traditional NMES system was used, this had little physiological impact on study participants, for example, there was only a $9 \%$ increase in energy expenditure above baseline values. The HbAlc assay measures chronic glycemia and is widely used to judge the adequacy of diabetes treatment. ${ }^{17} \mathrm{~A}$ related technology, described as 'high frequency NMES', was initially shown to have an effect on HbAlc. ${ }^{18}$ Further investigation did not appear to support this claim; however, it may have a beneficial effect on diabetic neuropathies. ${ }^{19} 20$ This 'high frequency NMES' appears to have negligible aerobic effect and so would probably have a different mechanism of action.

A small pilot study in patients with obesity $41 \pm$ $5.5 \mathrm{~kg} / \mathrm{m}^{2}$ suggested that the aerobic NMES system may be more suited to obese men than obese women. In a supervised session, the men $(n=3)$ could exercise at an average rate of $549 \mathrm{kcal}$ over $1 \mathrm{~h}$, including $30 \mathrm{~min}$ at $88 \pm 8 \%$ of their maximal heart rate and $59.9 \pm 9.6 \%$ of their maximal aerobic capacity $\mathrm{VO}_{2 \max }$. By contrast, the women could only exercise at a rate of $203 \mathrm{kcal}, 63 \pm 9 \%$ of their maximal heart rate and $41.1 \pm 12.0 \% \mathrm{VO}_{2 \max }$. All three men found the treatment 'highly acceptable', and the female participants had a more mixed reaction. Hence, it was decided to first assess the NMES system on men with diabetes only.

NMES systems that can deliver aerobic exercise could contribute towards the physical activity needs of patients with type 2 diabetes. An ideal system would not load the joints and could exercise with no gross hip or knee movements, it could be used even in the arthritic or those with peripheral neuropathies and could be used without supervision in the privacy of one's home while sitting watching television or reading. A foolproof system with rapid application that was acceptable and tolerable at therapeutic intensities may be appealing to both patients and clinicians. The objective of this pilot investigation was to see if (1) this new exercise modality was acceptable to and could be used by a sample of men with type 2 diabetes mellitus and (2) to assess if training with it over 8 weeks, unsupervised and at home, had an effect on haemoglobin Alc.

\section{PATIENTS AND METHODS}

Approval for the case series was given by the local university human research ethics committee.

\section{Recruitment and screening}

Participants were recruited by an advert placed in a local newspaper; this advert explicitly mentioned exercise: ... study 'investigating the benefits of a new form of exercise-can do watching TV, etc.'. It sought men with type 2 diabetes of less than 65 years who were diagnosed within the previous 5 years and were otherwise in good general health. Twenty-five potential participants (plus two people on behalf of relatives) contacted the researcher by phone for further information. All respondents were Caucasian. After telephone screening, see exclusion criteria in box 1, 12 subjects were invited into the university for further screening, information and to assess whether, after trying the device, they would like to participate in the study. Informed consent and permission from their personal doctor or endocrinologist was obtained. Where indicated an exercise cardiac stress test was completed prior to enrolment. One subject was unsure whether he wished to proceed. $\mathrm{He}$ immediately withdrew citing dislike of the sensations caused by the NMES. Of the remaining 11 participants, one withdrew due to repeated machine failures and two withdrew for personal reasons unrelated to the NMES. Hence, eight subjects were trained with the NMES. They had a body mass index of $32 \pm 55 \mathrm{~kg} / \mathrm{m}^{2}$ and were $53 \pm 8$ years old. There was no lifestyle or medication changes or significant weight fluctuation over the preceding 3 months. Three undertook no regular exercise, four exercised two-to-four times weekly and one exercised daily. During this introductory session, lasting 


\section{Box 1 Exclusion criteria}

Women

- >65 years

- Diagnosed as having diabetes more than 5 years ago

- Not within easy commute of the university

- Change of lifestyle in last 3 months (deemed by researcher to be relevant)

- Self-reported fluctuation in weight of over $3 \mathrm{~kg} / 6 \mathrm{lbs}$ in the past 3 months

- Other metabolic or hormonal disorder

- Blood clotting disorder

- Metal implants or cardiac pacemakers

- Inflammatory rheumatological disease

- Fractures during the past 6 months

- Lower extremity trauma during the past 6 months or currently experiencing related symptoms or receiving treatment

- Epilepsy

- Neoplastic disease

- Previous stroke

- Alzheimer's disease

- Serious respiratory disorder

- Parkinson's disease

- Major musculoskeletal disease/disorders

- Medically diagnosed osteoporosis

- Uncontrolled hypertension

- Previous myocardial infarction

- Cardiac arrhythmias

- Use of $\beta$ blockers

- Skin condition that may interfere with the delivery of the NMES

- Addicted to alcohol or drugs that interfere with the neuromuscular system

- Using insulin/injections of any kind

- Any other condition or history that the investigator considers might increase the risk to the individual or interfere with the study or the evaluation of data

15-30 min, all participants reached a heart rate of 120 beats per minute or more. This was assessed by palpation. All expressed confidence that they could apply the electrodes and control the stimulation without supervision.

\section{Intervention}

The eight participants were asked to train with the NMES, unsupervised, at home, for $45 \mathrm{~min}$ to $1 \mathrm{~h}$, six evenings a week, for eight weeks. All patients received at least 6-weeks training. (One patient was measured in the seventh week due to unexpected and unavoidable foreign travel. One missed several days training in the last week due to NMES unit failure.) Self-reported compliance was at least five times per week on average for all participants. The NMES unit had a built-in crude compliance monitor that would be supportive of the participants' stated usage levels. Whenever possible, they were instructed to train after their evening meal and not to eat prior to bed. There were no other dietary restrictions, lifestyle changes or medication changes required.
Technical description of the aerobic NMES stimulation system

The pulses were delivered through an array of eight large hydrogel electrodes, $17 \times 10.3 \mathrm{~cm}$, applied to the skin using two neoprene wrap garments, one applied to each thigh, see figure 1 . The electrodes were pre-wired and mounted for convenient rapid and correct application. In figure 1, the model's left leg is without the garment to illustrate electrode positioning. The basic NMES pulse pattern is a composite of four pulses shared between the electrode array (figure 2). Repeating the pulse pattern at $5 \mathrm{~Hz}$ induces a strong non-fused nontetanic contraction of the large muscle groups in the legs (quadriceps, hamstrings, gluteal and calf muscles).

The pulses were created using a programmable NT2010 research stimulator (Bio-Medical Research Ltd). This device allows for more complex pulses and pulse patterns than would be typical. A simplified schema of the pulse pattern is shown in figure 2. Pulses 1, 2 and 3 were $760 \mu$ s long; pulse 4 was $857 \mu$ s. All pulses are biphasic, symmetrical with an interphase delay of $100 \mu \mathrm{s}$. In turn, each pulse is divided into separate segments called timeslots. There were between three and five timeslots per pulse. For each of the 16 timeslots (in the first phase of the pulses), a subset of the electrode array has been designated as source or sink of the current. Furthermore, each timeslot can be allocated a percentage of the maximum current available - set by the user-controlled intensity button. For instance, for user comfort, the timeslot targeting vastus medialis is set at $60 \%$ of those targeting the proximal quadriceps. Using this system, the current densities 'seen' throughout the thigh can be optimised. The variability of this system enables the targeting of the gluteal and

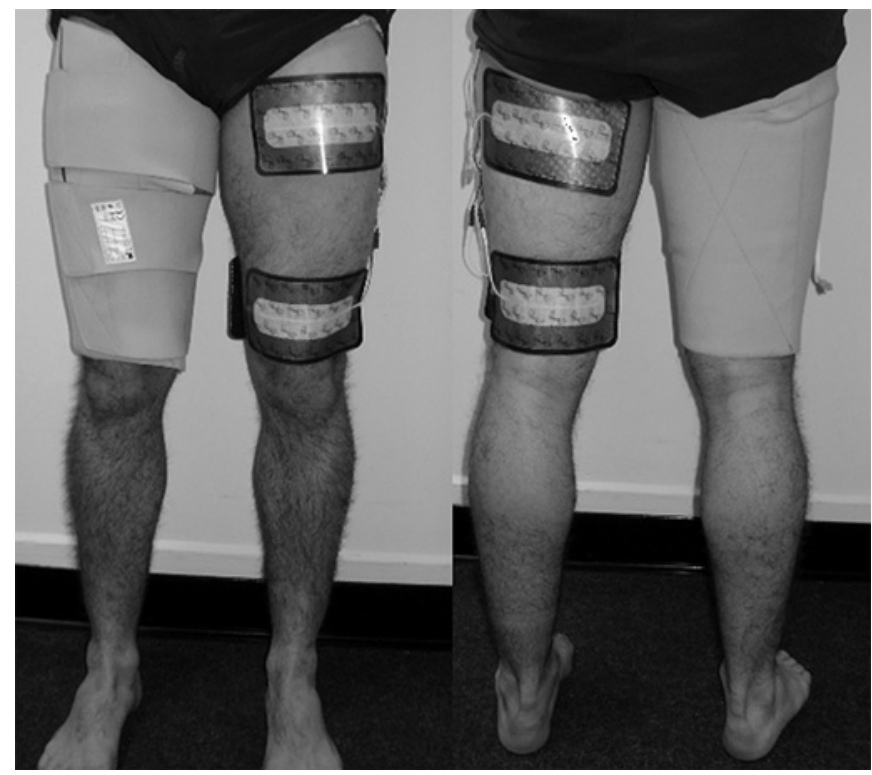

Figure 1 Four large hydrogel electrodes are applied to the skin of each thigh using a neoprene wrap. The electrodes were pre-wired and mounted inside the wrap. The model's left leg is without the garment to illustrated electrode positioning. 

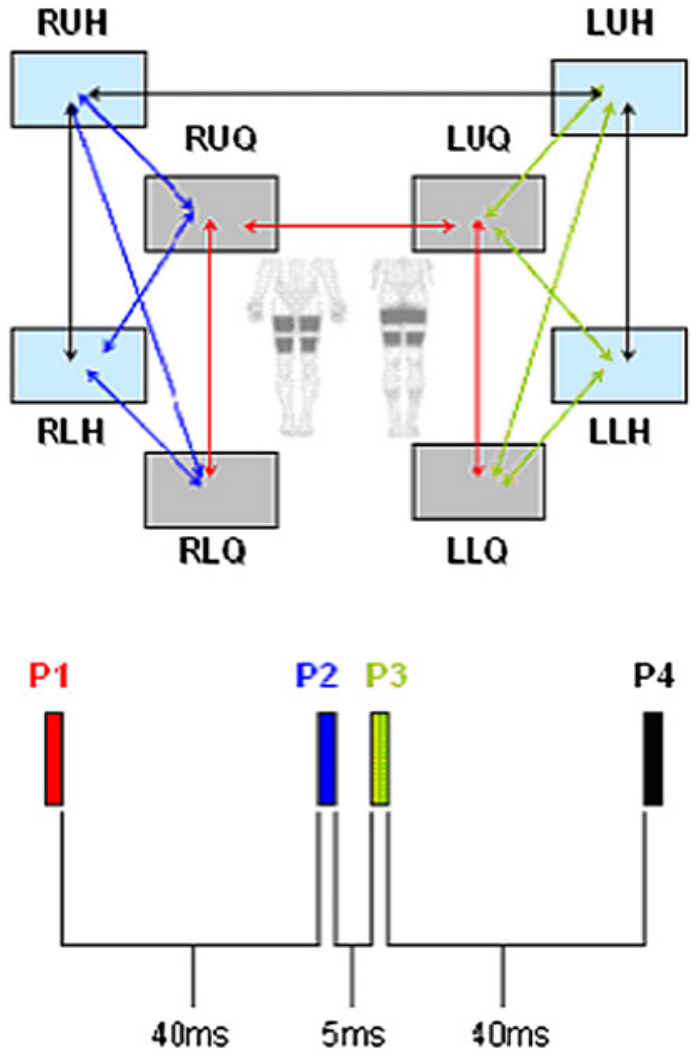

Figure 2 Simplified diagram of pulse pattern. Each pulse is represented by a different colour. The current passes between different sets of electrodes within each pulse. The electrodes are described by their position, that is, RUQ-right upper quadriceps electrode, $\mathrm{RUH}$-right upper hamstring,

RLQ-right lower quadriceps, etc. See also Figure 1 for picture of electrode positions. The pattern has four pulses; within this pattern, the pulses are separated by either 40 or $5 \mathrm{~ms}$. For aerobic effect, the pattern is repeated five times per second. Each pulse has a different function and is further subdivided into timeslots. Differing electrode combinations are active during separate timeslots of a given pulse, for example, pulse 1 , in red, is shared between the upper and lower quadriceps of both legs. By defining the timeslots, the upper quadriceps 'see' both a higher current intensity and a longer pulse duration than the lower electrodes. (This is not shown.) The second pulse, in blue, has five timeslots, and it targets the muscle bulk of the right leg. The third pulse, in green, follows $5 \mathrm{~ms}$ later; it is similar to the second pulse except it targets the muscle bulk of the left leg. Forty millisecond later, the fourth pulse, in black, spreads current between the hamstring electrodes.

calf muscles, even though there are no electrodes over these muscles, for example, the motor neurons supplying the calf muscle are stimulated as they traverse the upper leg.

\section{Measurements and statistical analysis}

Preweight and postweight, dual energy x-ray absorptiometry (DXA) scans (GE Healthcare, Waukesha, Wisconsin, USA) and venous blood samples, for HBA1c, were taken. The samples were refrigerated, then collected and analysed by an accredited laboratory (INAB; ISO 15189). Afterwards, the participants filled in a brief questionnaire given to them by the researcher. Two participants did not fill in the questionnaire, they were later phoned and their responses were noted. The questionnaire asked (1) What I liked most about it; (2) What I liked least about it; (3) As a form of exercise do you think it is suitable for diabetics? (4) If it brings about an improvement in diabetes but people still had to take medication would you recommend it? (5) If you needed to continue using it twice a week to maintain improvements in diabetes would you do it? (6) Suggestions and (7) Other comments.

The objective measures, see table 1 , were analysed by comparing before and after data using a two-tailed paired Student $\mathrm{t}$ test. As this was a pilot study on a novel therapy, there was no a priori power calculation. The questionnaire was qualitative by its nature. Questions 3-5 inclusive invited a yes/no response.

\section{RESULTS}

The results, displayed in table 1 , show mean HbAlc levels improved by $0.8 \pm 0.7 \%$ (mean \pm SD) from $7.4 \pm 1.3 \%$ prior to intervention to $6.6 \pm 1.0 \%$ postintervention $(p=0.01)$. The four participants with a starting HbAlc of less than seven improved from a mean of $6.5 \%-6.0 \%$ and those with a starting value of seven or more improved from a mean of $8.4 \%-7.3 \%$. Removing any one subject from the statistical analysis still leaves a statistically significant change in HbAlc of $p=0.03$ or less. The HbA1c for subject 8 was not available, however, furctosamine data were. This was converted to HbAlc using the formula \% HbAlc $=0.017 \times$ Fructosamine $+1.61 .^{21}$ Excluding this subject, the mean before $\% \mathrm{HbAlc}$ is $7.5 \pm 1.4 \%$, mean after $\% \mathrm{HbAlc}$ is $6.7 \pm 1.0 \%$, the change $-0.7 \pm 0.7 \%$ and $\mathrm{p}$ value is 0.03 . Changes in mass and lean mass were not statistically significant. Mean mass decreased by $0.7 \pm 2.7 \mathrm{~kg}(\mathrm{p}>0.05)$, while mean lean mass increased by $808 \pm 1762 \mathrm{~g}(\mathrm{p}>0.05)$.

From the questionnaire, all eight participants considered that the system suitable for people with type 2 diabetes mellitus would recommend it and would continue to use it twice a week 'to maintain improvements'. Three disliked the electrodes, one complained of hamstring cramps. What participants 'liked most about it' varied, 'improved energy levels', 'work up a good sweat-it could be done anywhere in the house, watching TV, etc.' 'feeling afterwards-like playing football when I was younger'.

\section{DISCUSSION}

The average improvement in HbAlc of $0.8 \pm 0.7 \%$ is clinically significant and compares well to other lifestyle interventions where an exercise effect of $0.62 \%$ improvement can be expected. ${ }^{22}$ The only intervention study in patients with type 2 diabetes with standard NMES $^{16}$ did 'not show any metabolic benefit'. Although it showed that the NMES 'mimics acute exercise... the magnitude of this change is too small to have any clinical 
Table 1 Preintervention and postintervention values for \%HbA1c, mass and lean mass

\begin{tabular}{|c|c|c|c|c|c|c|c|}
\hline & $\begin{array}{l}\% \mathrm{HbA1c} \\
\text { preintervention }\end{array}$ & $\begin{array}{l}\% \mathrm{HbA1c} \\
\text { postintervention }\end{array}$ & $\begin{array}{l}\% \text { HbA1c } \\
\text { change }\end{array}$ & $\begin{array}{l}\text { Mass }(\mathbf{k g}) \\
\text { preintervention }\end{array}$ & $\begin{array}{l}\text { Mass (kg) } \\
\text { postintervention }\end{array}$ & $\begin{array}{l}\text { Mass (kg) } \\
\text { change }\end{array}$ & $\begin{array}{l}\text { Lean }(g) \\
\text { change }\end{array}$ \\
\hline 1 & 6.1 & 6.1 & 0 & 115 & 110.6 & -4.4 & -442 \\
\hline 3 & 9.4 & 7.4 & -2 & 102.2 & 102.1 & -0.1 & 1073 \\
\hline 4 & 6.2 & 5.5 & -0.7 & 91 & 85.6 & -5.4 & -1939 \\
\hline 5 & 9.5 & 8.5 & -1 & 93 & 94.8 & 1.8 & 1249 \\
\hline 8 & $6.9^{\star}$ & $5.7^{\star}$ & -1.2 & 115.7 & 116.9 & 1.2 & 2411 \\
\hline Mean $\pm S D$ & $7.4 \pm 1.3$ & $6.6 \pm 1.0$ & $-0.8 \pm 0.7 \dagger$ & $103.8 \pm 22.0$ & $103.0 \pm 22.6$ & $-0.7 \pm 2.7 \ddagger$ & $808 \pm 1762 \S$ \\
\hline
\end{tabular}

benefit'. The main difference with this aerobic system is that the NMES can induce large metabolic demand. ${ }^{1-5}$ The full extent of the improvement may not be captured by the HbAlc as the intervention period was only 8 weeks in duration. The slight mass decrease $(0.7 \pm 2.7 \mathrm{~kg})$ makes it unlikely that dieting or other lifestyle modifications were the driver of the HbAlc changes. Several participants commented that they liked not being asked to lose weight or make other lifestyle modifications. However, in the formal debriefing afterwards, two participants reported changes in diet. Subject 1 said he ate fewer home-baked delicacies than usual. Subject 4 reduced his alcohol intake (from a reported 'can of beer most evenings'). Removing subjects 1 and 4 from the analysis still leaves a statistical significance of $\mathrm{p}<0.02$ for changes in HbA1c.

The changes in lean mass are not statistically significant and vary considerably between subjects (mean \pm SD: $0.8 \pm 1.7 \mathrm{~kg}$ ). This may be partly due to the small numbers and the test-retest reliability of the system. GE Healthcare report that for the system used (Lunar iDXA), 'statistically $68 \%$ of repeat scans fall within 1 SD $\ldots \pm 310 \mathrm{~g}$ lean mass'. Presumably, this reliability would further deteriorate when separated by 2 months and subjects having different hydration levels and mass, etc. Surprisingly, the only subject to increase his HbAlc put on the most lean mass according to the DXA scan.

All participants found the treatment acceptable and, tellingly, all would like to be contacted for further research on it. All reported that they would like to continue using the device 'twice a week to maintain improvements'. While not explicitly sought on the questionnaire, the participants would not wish to continue exercising with it six times weekly. The results of the questionnaire were uniformly positive. However, this probably partly reflects selection bias and their participation in the study. They were a pro-active group who volunteered for an exercise study.

The sensation of exercising without volitional contractions is, at first, strange to many. Accommodation and habituation to the stimulation proved to be important both within and between sessions. This habituation process is typical of NMES. ${ }^{23}$ All participants had shown that they could use the system at a level that induced a heart rate of at least $120 \mathrm{bpm}$ in the observed introductory session. All reported that they could use it at levels that made them sweaty and breathless (affects speech). This probably compares well to actual practice in the target population. In a study of over 400 patients with type 2 diabetes mellitus, Thomas reports that 'only $9 \%$ of these patients exercised sufficiently to achieve a large change in heart rate or breathing'. ${ }^{24}$

Intriguingly, there may be particular advantages of this exercise modality-especially when used in addition to voluntary exercise. NMES is thought to preferentially recruit different motor units in position and in type. ${ }^{25}$ Kimura $e t a l^{15}$ has argued the potential benefits in type 2 diabetes of a higher proportion of type IIb fibres being exercised. If a person both walks and uses this modality, then there will be a different fibre mix recruited by each exercise type and the total proportion of fibres exercised will be higher. Furthermore, traditional NMES techniques are known to elicit different metabolic adaptations compared with voluntary exercise. Gondin et $a l^{26}$ showed a significant shift in muscle fibre type from myosin heavy chain (MHC) MHC-2X towards MHC-2A and MHC-1 and evidence of a muscle fibre shift towards oxidative metabolism, that is, a glycolytic-to-oxidative shift in the metabolic profile. It is unclear if the new NMES techniques will induce a similar metabolic effect. However, strength increases in addition to increases in aerobic capacity have been noted. ${ }^{3}$ Exercising with this system appears to preferentially use carbohydrate as a substrate, that is, exercise at a very high respiratory quotient. This has been documented in a healthy subject $^{127}$ but has also been noted in a group of nine patients with morbid obesity (unpublished data). Consistent with this is high lactate levels, up to $15.3 \mathrm{mmol} / \mathrm{L}$, that have been recorded. ${ }^{1}$ This has also been noted with traditional NMES techniques. ${ }^{28}$ The 
aerobic NMES exercise appears to have a metabolic profile more consistent with vigorous voluntary exercise-an exercise intensity that many with type 2 diabetes may not engage in sufficiently. ${ }^{24}$ The role of lactate may be significant; Sola-Penna ${ }^{29}$ argues that lactate may no longer be considered a 'worthless metabolite' but 'a regulatory molecule that modulates the integration of metabolism' including glucose.

The positive reaction from the participants and the improvements in HbAlc gives us confidence that the system may have a role to play in treating some of those with type 2 diabetes. It may be particularly suited to those with barriers to voluntary exercise, for example, the high proportion of patients with arthritis-attributable activity limitation. ${ }^{11}{ }^{12}$ There are no gross movements in the hips or the knees, excepting some patellar gliding, and it does not load the joints. Some may like to use it simply out of preference over voluntary exercise or for its convenience or because it is a 'passive' exercise and can be done in private while watching television. In the unfit, there appears to be no minimum threshold of exercise intensity to reap benefits. ${ }^{30}$ This may also apply to this system of exercise. Women with obesity seem less likely to exercise with this at levels greater than $40 \%$ of VO2 reserve (unpublished data). Perhaps, the system could be used at lesser intensity for longer. US adults still watch an average of $153 \mathrm{~h}$ of television per month. ${ }^{31}$ Total sitting time is associated with weight gain independently of leisure time physical activity ${ }^{32}$ and television watching is also independently associated with increased risk of type 2 diabetes. ${ }^{33}$ Some of this sitting time could become physical activity. A fit person using this NMES system while watching television for $6 \mathrm{~h}$ burnt over $2200 \mathrm{kcal} .{ }^{1} \mathrm{~A}$ greater understanding of the mechanisms of action may help further improve the efficacy of the treatment. For instance, the present system is optimised to maximise oxygen consumption; however, preliminary testing suggests that it can be further modified to give a higher lactate output for a given oxygen consumption.

Further investigation of the system on a larger group, with a control arm and double blinding, seems warranted. Of particular interest would be a study of those who have significant barriers to voluntary exercise but can exercise with this system. A greater understanding of the metabolism of this exercise modality may suggest further improvements to the system. The authors have also hypothesised that the system may have a potential application as part of a novel accelerated programme to help prevent the progression of diabetes/prediabetes. ${ }^{27}$

This was a small pilot study undertaken in a group of pro-active enthusiastic men with type 2 diabetes. While the results are promising, it was not a randomised controlled trial, and the sample was not representative of the 366 million $^{34}$ people with diabetes.

\section{CONCLUSION}

The results of this pilot investigation are encouraging and suggest, for the first time, a viable aerobic exercise alternative that may be suitable for some with type 2 diabetes who cannot or will not engage in voluntary exercise.

Acknowledgements The authors would like to express their grateful appreciation for the encouragement and financial/material support from Enterprise Ireland, an Irish governmental body, and Bio-Medical Research (BMR) Ltd. The corresponding author worked with BMR Ltd more than 5 years ago and has a minor holding in the company. BMR Ltd supplied the NMES units.

Contributors LC developed the technology (with BMR Ltd who designed and provided the stimulation platform and software) and parameters, tested the subjects and wrote the paper. BC was responsible for obtaining institutional ethics clearance, contributed to the study design, assisted with the study's implementation and revised the paper. Both authors approved the final version.

Funding Enterprise Ireland. (An Irish governmental body). Funding for a university-based project investigating NMES.

\section{Competing interests None.}

Ethics approval Ethics approval was provided by UCD Human Ethics Committee.

Provenance and peer review Not commissioned; externally peer reviewed.

Data sharing statement The NMES parameters used to induce a large aerobic effect are non-standard and quite intricate. The normal NMES nomenclature and descriptions are not applicable to much of it. A lot of the detail has been included in the article but we invite research groups to contact us for full details, explanation, etc.

\section{REFERENCES}

1. Crowe L, Caulfield B. Pushing out the limits of electrical stimulation. A case study in the aggressive use of an alternative to voluntary exercise. BMJ Case Reports 2011; doi:10.1136/bcr.06.2011.4343

2. Crognale D, Crowe L, Devito G, et al. Neuro-muscular electrical stimulation training enhances maximal aerobic capacity in healthy physically active adults. Conf Proc IEEE Eng Med Biol Soc 2009:2137-40.

3. Banerjee P, Caulfield B, Crowe L, et al. Prolonged electrical muscle stimulation exercise improves strength, peak VO2, and exercise capacity in patients with stable chronic heart failure. J Card Fail 2009;15:319-26.

4. Banerjee $\mathrm{P}$, Caulfield B, Crowe $\mathrm{L}$, et al. Prolonged electrical muscle stimulation exercise improves strength and aerobic capacity in healthy sedentary adults. J Appl Physiol 2005;99:2307-11.

5. Caulfield B, Crowe L, Minogue C, et al. The use of electrical muscle stimulation to elicit a cardiovascular exercise response without joint loading: a case study. J Exerc Physiol 2004;7:84-8.

6. Alberti KG, Zimmet P, Shaw J. International Diabetes Federation: a consensus on Type 2 diabetes prevention. Diabet Med 2007;24:451-63.

7. Nathan DM, Buse JB, Davidson MB, et al. Medical management of hyperglycaemia in type 2 diabetes mellitus: a consensus algorithm for the initiation and adjustment of therapy: a consensus statement from the American Diabetes Association and the European Association for the Study of Diabetes. Diabetologia 2009; 52:17-30.

8. Korkiakangas EE, Alahuhta MA, Laitinen JH. Barriers to regular exercise among adults at high risk or diagnosed with type 2 diabetes: a systematic review. Health Promot Int 2009;24:416-27.

9. Ball K, Crawford D, Owen N. Too fat to exercise? Obesity as a barrier to physical activity. Aust N Z J Public Health 2000;24:331-3.

10. Hootman JM, Macera CA, Ham SA, et al. Physical activity levels among the general US adult population and in adults with and without arthritis. Arthritis Rheum 2003;49:129-35.

11. Centers for Disease Control and Prevention. Prevalence of doctordiagnosed arthritis and arthritis-attributable activity limitation-United States, 2007-2009. MMWR Morb Mortal Wkly Rep 2010;59:1261-5. http://www.cdc.gov/mmwr/preview/mmwrhtml/mm5939a1.htm (accessed Dec 2011).

12. Bolen J, Hootman J, Helmick CG, et al. Arthritis as a potential barrier to physical activity among adults with diabetes-United States, 2005 and 2007. National Center for Chronic Disease Prevention and Health Promotion, CDC. MMWR Morb Mortal Wkly Rep 2008;57:486-9. 
http://www.cdc.gov/mmwr/preview/mmwrhtml/mm5718a3.htm (accessed 15 Jun 2010).

13. Sallis JF, Hovell MF. Determinants of exercise behavior. Exerc Sport Sci Rev 1990;18:307-30.

14. Varo JJ, Martinez-Gonzalez MA, De Irala-Estevez J, et al. Distribution and determinants of sedentary lifestyles in the European Union. Int $J$ Epidemiol 2003;32:138-46.

15. Kimura $\mathrm{T}$, Matsumoto $\mathrm{K}$, Kameda $\mathrm{N}$, et al. Percutaneous electrical muscle stimulation attenuates postprandial hyperglycemia in obese and pre-obese Japanese men. Int J Sports Health Sci 2010;8:1-6.

16. Poole RB, Harrold CP, Burridge $\mathrm{JH}$, et al. Electrical muscle stimulation acutely mimics exercise in neurologically intact individuals but has limited clinical benefits in patients with type 2 diabetes. Diabetes Obes Metab 2005;7:344-51.

17. Nathan DM, Kuenen J, Borg R, et al. Translating the A1C assay into estimated average glucose values. Diabetes Care 2008;31:1473-8.

18. Rose $B$, Lankisch $M$, Herder $C$, et al. Beneficial effects of external muscle stimulation on glycaemic control in patients with type 2 diabetes. Exp Clin Endocrinol Diabetes 2008;116:577-81.

19. Kempf K, Martin S. Hochfrequente externe Muskelstimulation verbessert signifikant die Symptome der diabetischen Neuropathie. gbo Zurich: Medizintechnik, 2010.

20. Kempf K, Martin S. High-frequency external muscle stimulation in the relief of symptomatic diabetic neuropathy. Diabetes Stoffw Herz 2010;19:3-9.

21. Cohen RM. Holmes YR, Chenier TC, et al. Discordance between $\mathrm{HbA1c}$ and fructosamine: evidence for a glycosylation gap and its relation to diabetic nephropathy. Diabetes Care 2003;26:163-7.

22. Thomas DE, Elliott EJ, Naughton GA. Exercise for type 2 diabetes mellitus. Cochrane Database Syst Rev 2006;(3):CD002968.

23. Alon G, Smith GV. Tolerance and conditioning to neuromuscular electrical stimulation within and between sessions and gender. $J$ Sports Sci Med 2005;4:395-405.
24. Thomas N, Alder E, Leese G. Barriers to physical activity in patients with diabetes. Postgrad Med J 2004;80:287-91.

25. Gregory CM, Bickel CS. Recruitment patterns in human skeletal muscle during electrical stimulation. Phys Ther 2005;85:358-64.

26. Gondin J, Brocca L, Bellinzona E, et al. Neuromuscular electrical stimulation training induces atypical adaptations of the human skeletal muscle phenotype: a functional and proteomic analysis. $J$ Appl Physiol 2011:110:433-50.

27. Crowe L, Caulfield B. Towards creating a super-stimulus to normalise glucose metabolism in the pre-diabetic; a case study in the feast-famine and activity-rest cycle. BMJ Case Reports 2012; doi:10.1136/bcr.03.2011.3939

28. Hamada T, Hayashi T, Kimura T, et al. Electrical stimulation of human lower extremities enhances energy consumption, carbohydrate oxidation, and whole body glucose uptake. J Appl Physiol 2004:96:911-16.

29. Sola-Penna M. Metabolic regulation by lactate. Life $2008 ; 60$ : 605-8.

30. Swain D, Franklin B. VO2 reserve and the minimal intensity for improving cardiorespiratory fitness. Med Sci Sports Exerc 2002;34:152-7.

31. The Three Screen Report. First Quarter. The Nielson Company. 2009. http://www.nielsen.com/content/corporate/us/en/search.html? $\mathrm{q}=$ Three+screen+report (accessed Sept 2011).

32. Brown W, Williams L, Ford J, et al. Identifying the energy gap: magnitude and determinants of 5-year weight gain in midage women. Obes Res 2005;13:1431-41.

33. Hu FB, Li TY, Colditz GA, et al. Television watching and other sedentary behaviors in relation to risk of obesity and type 2 diabetes mellitus in women. JAMA 2003;289:1785-91.

34. International Diabetes Federation. Diabetes Atlas. http://www.idf.org/ diabetesatlas/5e/foreword (accessed Dec 2011). 